\title{
A statistical study of the motion of pulsating aurora patches: using the THEMIS All-Sky Imager
}

\author{
Bing Yang, Eric Donovan, Jun Liang, and Emma Spanswick \\ Department of Physics and Astronomy, University of Calgary, Calgary, Alberta, Canada \\ Correspondence to: Bing Yang (biyang@ucalgary.ca)
}

Received: 15 November 2016 - Revised: 11 January 2017 - Accepted: 12 January 2017 - Published: 8 February 2017

\begin{abstract}
Previous studies of the motion of patches that comprise patchy pulsating aurora (PPA) have been based on a limited number of events. In this study, we use a large database of PPA patches obtained from the THEMIS all-sky imager at Gillam (66.18 ${ }^{\circ}$ magnetic latitude, $332.78^{\circ}$ magnetic longitude) between May 2006 and July 2013 to explore the velocity of the PPA patches. Our results show that PPA patches mainly drift eastward after midnight and westward before midnight. In addition, we found that patch velocities are in the expected range of convection given the magnetic latitude and that the velocities do not seem to depend on auroral electrojet (AE) index. The results suggest that the drifts of auroral patches could be a proxy for the ionospheric convection, and possibly provide a convenient and accurate method to remotely sense the magnetospheric convection.
\end{abstract}

Keywords. Magnetospheric physics (auroral phenomena; magnetosphere-ionosphere interactions; plasma convection)

\section{Introduction}

An aurora is a common ionospheric phenomenon, which is a photon emission in Earth's upper atmosphere caused by the energetic particles precipitating from the magnetosphere. Patchy pulsating aurora (PPA), a common form of aurora and specifically caused by precipitating magnetospheric electrons, occurs mostly in the morning sector near the equatorward part of the auroral oval (Siren, 1975). PPA is often excited by electrons with energy of a few $\mathrm{keV}$ to tens of keV (Bryant et al., 1971; Sandahl et al., 1980). PPA patches are normally tens to hundreds of kilometers in extent and turn on and off quasi-periodically. The on-off cycle is most frequently between 1 and $40 \mathrm{~s}$ (Johnstone, 1978; Davidson, 1990). It is now known that the precipitating process of elec- trons is driven by wave-particle interactions (e.g. Nishimura et al., 2010), but whatever determines the pulsating period and the shape of patches is not well understood yet.

One typical characteristic of the morphology of PPA is that many PPA patches have the tendency to maintain their geometries for minutes or even longer (Royrvik and Davis, 1977). These geometries reflect either the spatial distribution of the source plasma or the spatial distribution of the conditions which cause the precipitation. Thus, these shapes could be used to investigate the physical processes creating the pulsations (Johnstone, 1978). Since any structure in a hot magnetospheric plasma would be rapidly smeared out by gradient and curvature drift, previous studies have suggested that the structure of PPA patches reflects the structure in the cold plasma near the equator in the magnetosphere (Oguti et al., 1976; Johnstone, 1978; Nakamura and Oguti, 1987; Davidson, 1990).

Another key aspect of PPA is the motion of the patches. Using data from all-sky cameras, Davis (1962, 1971) suggested that the motion of the auroral structures could be caused by convection drift in the magnetosphere. However, the author used a frame rate and integration time of $1 \mathrm{~min}$, so the patches are likely to have moved while each image was taken. Using data from the Scandinavian Twin Auroral Radar Experiment (STARE; Greenwald et al., 1978) and simultaneously obtained sequences of auroral images, Scourfield (1983) argued, on the basis of one single event, that PPA patches move with the $E \times B$ drift velocity. Moreover, Yang et al. (2015) used five events where a PPA patch was observed by an All-Sky Imager (ASI) in a region where there were simultaneous SuperDARN echoes, and found the velocities of PPA patches are close to SuperDARN-inferred ionospheric convection velocities. They suggested that the motion 

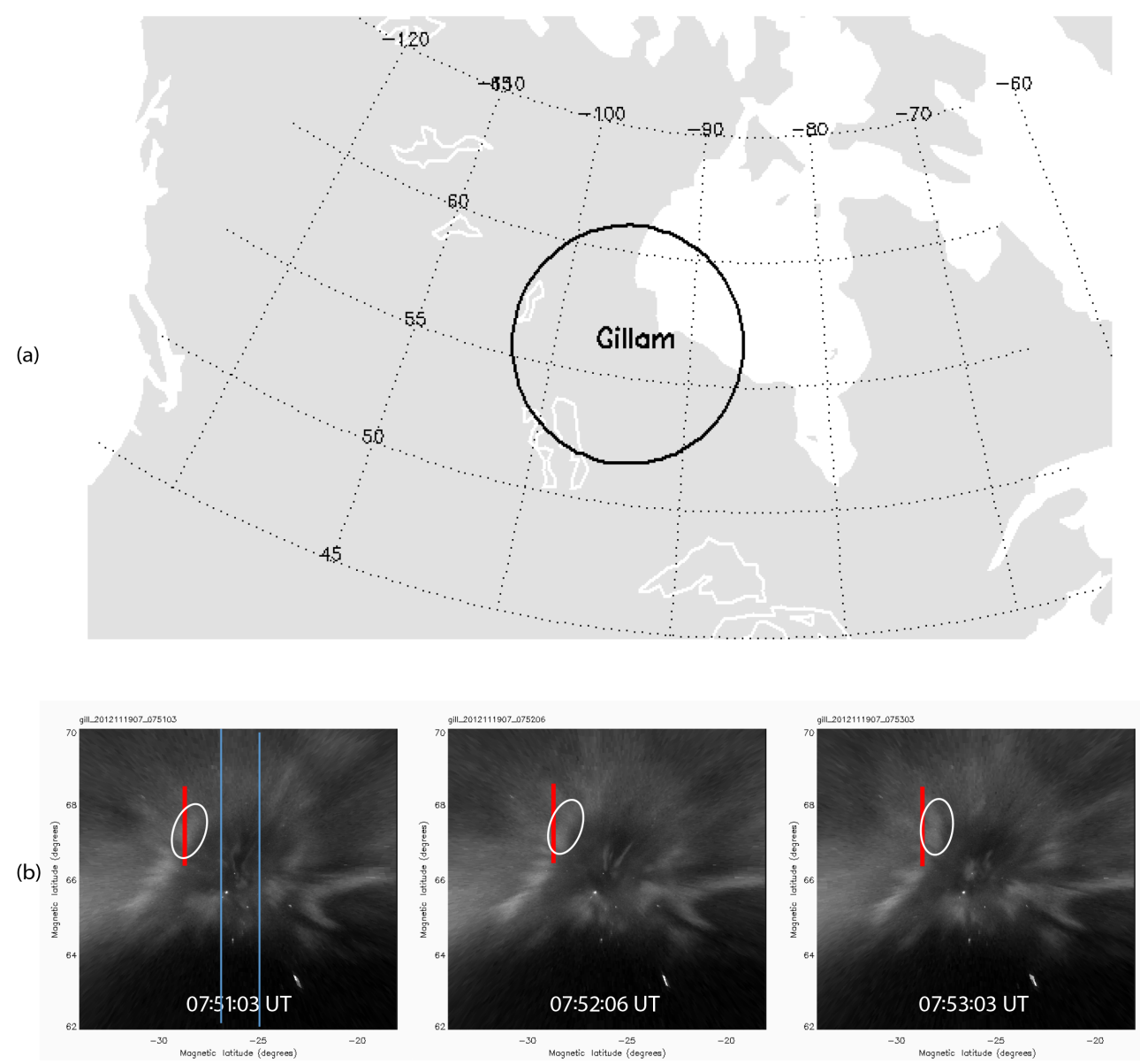

Figure 1. (a) Map of the location of THEMIS-ASI Gillam station. The black circle gives the coverage of the station. (b) Three all-sky images at an interval of about $1 \mathrm{~min}$. The white circle indicates a PPA patch. The red line as a reference gives the location of the patch at 07:51:03 UT.

of PPA patches could be used to remotely sense the ionospheric convection.

All the results above suggest that the motion of PPA patches is $E \times B$ motion and so reflects ionospheric convection. They support the hypothesis that the structure of PPA patches reflects cold plasma structures in the magnetosphere, with energy low enough so that the curvature and gradient drift can be neglected compared to the $E \times B$ drift (or convection). However, in total, studies to date reflect less than 10 events and further investigation needs to be done so that we can conclusively say that PPA patch motion is, or is not, convection. In this paper, we contribute to this line of research with results from a survey of PPA motion from hundreds of events.

The All-Sky Imager (ASI) array deployed as part of the NASA Time History of Events and Macroscale Interactions during Substorms (THEMIS) mission produces data suitable for our objectives. For our study, we carry out a survey using data from THEMIS-ASI Gillam station due to its magnetic latitude, which affords frequent PPA observations, and the generally high quality of the data. We identified over 300
PPA events during the period from May 2006 until July 2013 from which the velocity of the patches can be manually inferred.

\section{Data}

THEMIS-ASI includes 21 white-light ASIs distributed over North American continent from Canada to Alaska (Angelopouos, 2008; Donovan et al., 2006; Mende et al., 2008). Each imager produces a $256 \times 256 \times 16$ bit image every $3 \mathrm{~s}$ with $\sim 1 \mathrm{~km}$ spatial resolution near zenith at $110 \mathrm{~km}$ altitude, which is ideal for investigating the motion of PPA patches. The latitudinal coverage of each imager is $\sim 9^{\circ}$ and the longitude coverage is slightly more than $1 \mathrm{~h}$ magnetic local time (MLT). The Gillam station is at $56.4^{\circ}$ geographic latitude and $-96.7^{\circ}$ geographic longitude $\left(66.18^{\circ}\right.$ magnetic latitude, $332.78^{\circ}$ magnetic longitude), as shown in Fig. 1a. Figure $1 \mathrm{~b}$ gives an example of the motion of a PPA patch observed in the Gillam data on 19 November 2012 (please also see the supplement movie). The red line indicates the location of the 

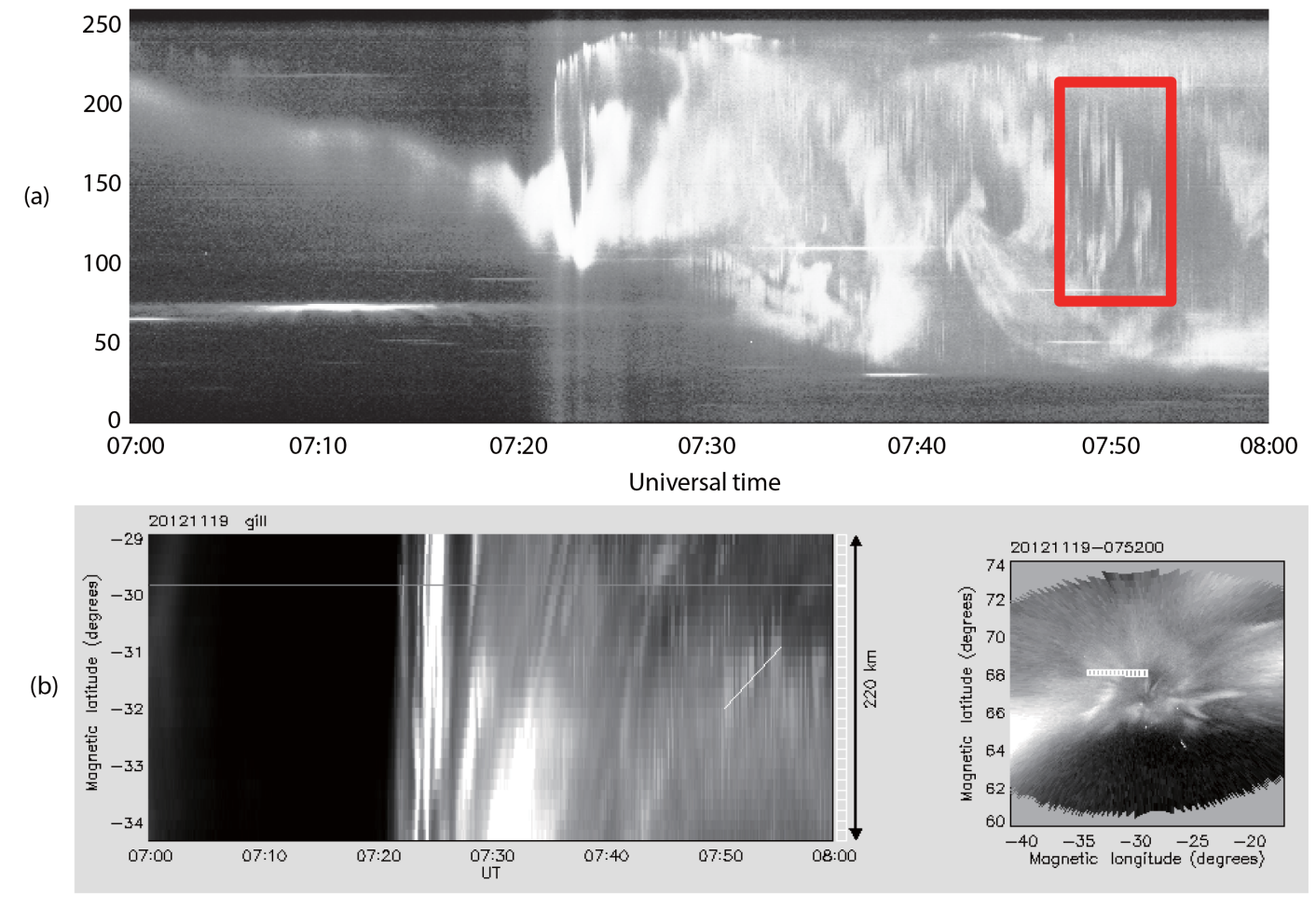

Figure 2. (a) A $1 \mathrm{~h}$ keogram from 07:00 to 08:00 UT on 19 November 2012. The horizontal axis is universal time and the vertical axis is the number of pixels. The red frame indicates where the PPA patch is identified. (b) A 1 h ewogram from 07:00 to 08:00 UT on 19 November 2012. The horizontal axis in the left panel is the universal time and the vertical axis in the left panel is the magnetic longitude. The right panel is an all-sky image in geomagnetic coordinate. The white line in the left panel indicates the motion of the identified PPA patch and that the white rectangle in the right panel indicates the pixels used to construct the ewogram.

patch at 07:51:03 UT. We can see the patch moves slightly to the east in those $2 \mathrm{~min}$.

We identified each event of PPA from the $1 \mathrm{~h}$ keograms that are commonly used to summarize the auroral observations. Keograms are constructed by extracting columns (as shown between the two blue lines in Fig. 1b) at the center of individual all sky images and putting them side by side. Figure 2a gives an example of a keogram from 07:00 to $08: 00$ UT on 19 November 2012, in which the $x$ axis is the universal time and the $y$ axis is the number of pixels. As images from THEMIS ASI are created every $3 \mathrm{~s}$ and PPA exhibits a quasi-periodic intensity variation, the pulsation feature of patches is clearly shown by the intermittent lines in the area of the keograms indicated by the red rectangle in Fig. 2a. In this study, $357 \mathrm{~h}$ with PPA events have been identified from $1 \mathrm{~h}$ keograms during May 2006 to July 2013.

To investigate the azimuthal motion of PPA patches, we constructed hourly east-west-o-grams (ewograms) for each hour we have identified PPA events from keograms. To construct the ewograms, we first transferred the images from THEMIS ASI in geographic coordinates into Altitude-Adjusted Corrected Geomagnetic (AACGM) coordinates (Baker and Wing, 1989) assuming a $110 \mathrm{~km}$ emission height; we then extracted a rectangle of pixels contain- ing PPA patches in the azimuthal direction from individual ASI images in the geomagnetic coordinate and put them side by side in $1 \mathrm{~h}$ series. Figure $2 \mathrm{~b}$ gives an example of how we constructed the ewograms. The left panel of Fig. $2 \mathrm{~b}$ is a $1 \mathrm{~h}$ ewogram from 07:00 to 08:00 UT on 19 November 2012, in which the horizontal axis is universal time and the vertical axis is magnetic longitude. The right panel of Fig. $2 b$ shows one ASI image with visible PPA patches in geomagnetic coordinates. The white rectangle in the right panel indicates the pixels we choose to construct the ewogram. The azimuthal (longitudinal) motion of patches is indicated by the white line in the left panel. The velocity of the patch is derived from the slope of the curve traced out by the patch. The slope is manually traced through the center of the patch and the typical error in estimating the velocity is $\sim \pm 10 \%$. Positive and negative slopes indicate eastward and westward PPA patch motion, respectively. To calculate the PPA velocity, we convert the slope in the ewogram to $\mathrm{m} \mathrm{s}^{-1}$. The PPA patch in Fig. $2 \mathrm{~b}$, which appeared at Gillam station from 07:51 to 07:53 UT, is moving $279 \mathrm{~m} \mathrm{~s}^{-1}$ to the east. The PPA motion is relative to the motion of the Earth, so the velocity is in the corotating frame of reference. 
(a)

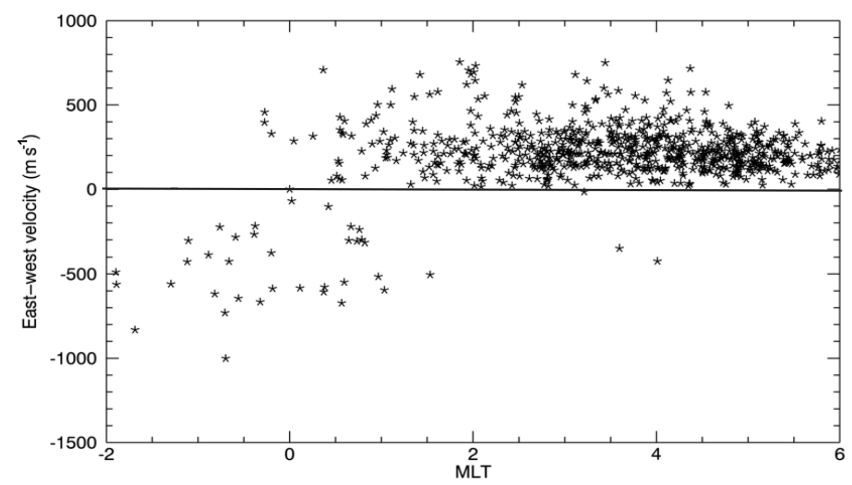

(b)

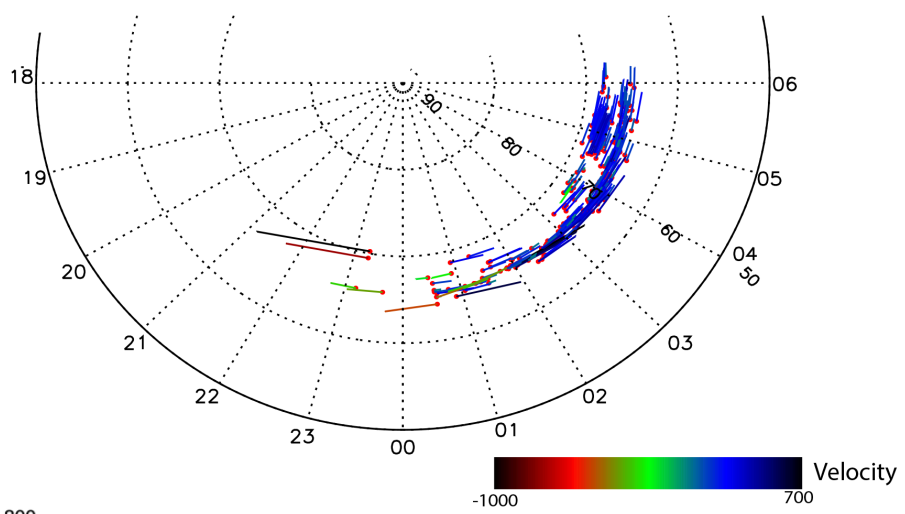

(c)

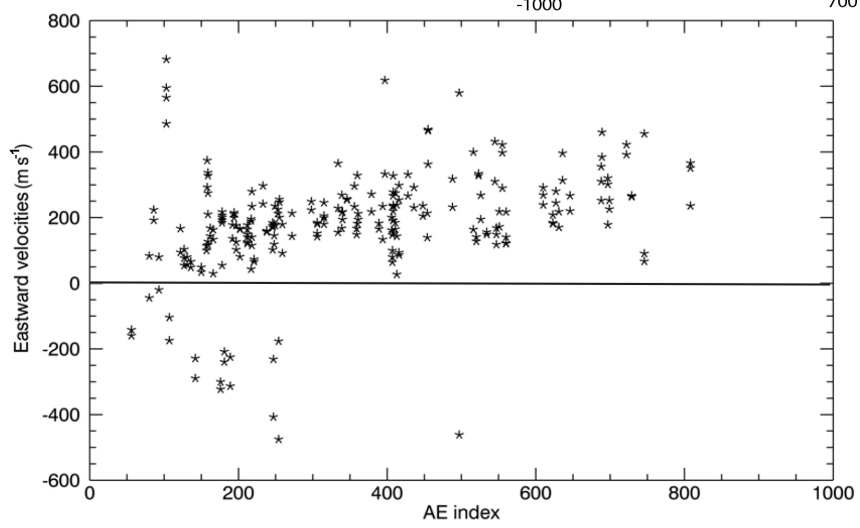

Figure 3. (a) Distribution of the patches' azimuthal velocities. The horizontal axis is the magnetic local time. The vertical axis is the eastward velocity in $\mathrm{m} \mathrm{s}^{-1}$. (b) Map of velocities of all the patches in the magnetic local time and magnetic latitude. (c) Distribution of patch velocities with respect to $\mathrm{AE}$ index.

\section{Results}

Figure 3a gives the distribution of eastward velocities of all PPA patches with respect to MLT. It shows that PPA patches mainly drift eastward after midnight and westward before midnight. Most patches in the morning sector are within the velocity range of 200 to $300 \mathrm{~m} \mathrm{~s}^{-1}$ (again, with respect to the ground).

Figure $3 b$ gives a distribution map with respect to MLT and magnetic latitude of the PPA patch velocities. There is a shear of velocities spread around 1.6 MLT. The same phenomenon is echoed by the convection map during substorms, illustrated, for example, by Weimer (1999), in which the sep- aration of two convection cells is not at midnight but moves to the morning sector. Nakamura and Oguti (1987) and Jones et al. (2011) found a similar result as well, and the former suggested that the shear is due to Harang discontinuity (Erickson et al., 1991).

Figure $3 \mathrm{c}$ depicts the relationship between the east-west velocities and auroral electrojet (AE) index for the patch events. Since we define one slope (velocity) for each patch, so the determined velocity represents the average speed of the patch during its lifetime within the imager field of view (typically a few tens of minutes). The AE value for each patch event used here is found from hourly AE index using linear interpolation. The result shows a weak correlation be- 
(a)
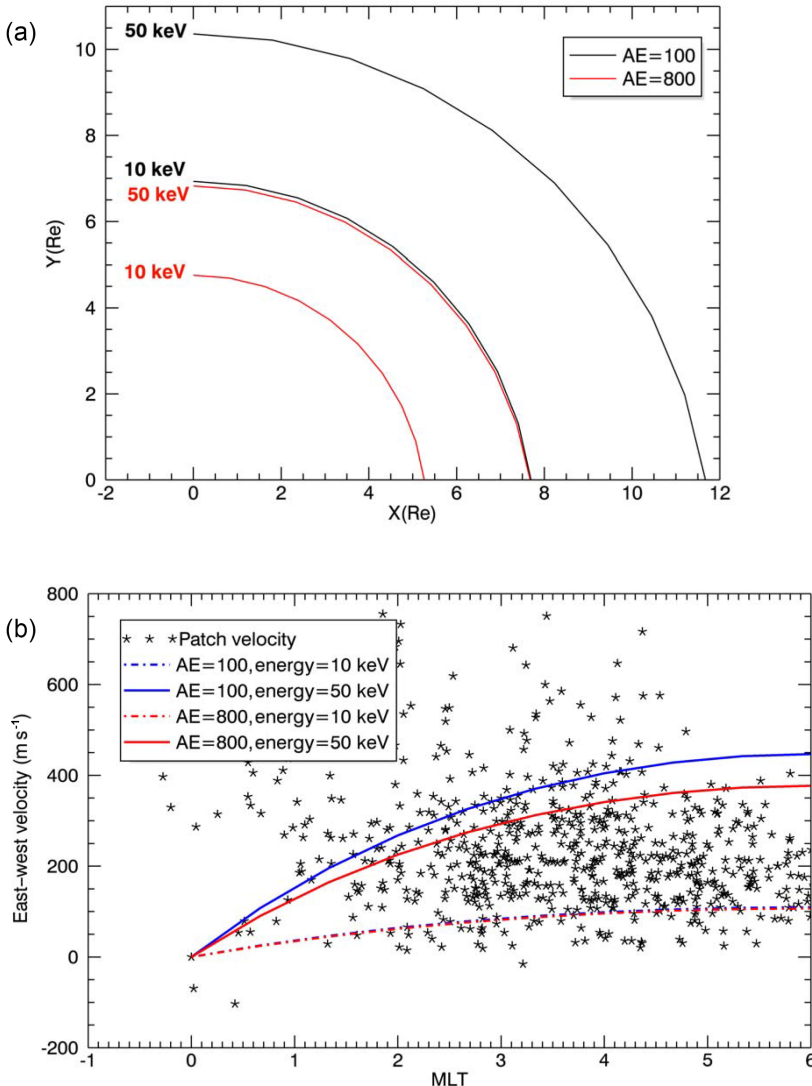

Figure 4. (a) The inner boundaries of $45^{\circ}$ pitch angle electrons with energy of 10 and $50 \mathrm{keV}$ on the equatorial plane. Red and black represent boundaries for $\mathrm{AE}=800$ and $\mathrm{AE}=100$, respectively. (b) Distribution of MLT versus patch eastward velocity for the events with convection velocities inferred from the electric field model at the inner boundaries of electron plasma sheet with each AE index and electron energy. The convection velocities have been mapped into the ionosphere and the corotation has been subtracted.

tween patch eastward velocities and $\mathrm{AE}$ index (correlation value $=0.36$ ), which at first appears to be at odds with the idea that patches are moving with convection, as we know overall convection strength is closely related to $\mathrm{AE}$ (Weimer et al., 1990). We have also examined the relationship between PPA velocities and instantaneous solar wind parameters, i.e. magnetic field magnitude, electric field and plasma flow speed. There is no strong relationship between PPA velocities and these parameters as well.

\section{Discussion}

The AE index is found to correlate with the solar wind electric field for quiet and moderately strong solar wind conditions (Weimer et al., 1990). Their results also show that the cross-polar-cap potential and in turn the overall convection at $\mathrm{AE}=800$ would be at least 3 times the convection veloc- ity at $\mathrm{AE}=100$. However, our observations indicate a weak (if any) relationship between patch velocities and AE. To explain this discrepancy, we can consider the physical nature of PPA. PPA is widely believed to be excited by the waveparticle interactions between chorus waves and energetic electrons in the equatorial magnetosphere (e.g. Nishimura et al., 2010). The growth of whistler-mode chorus waves depends on two factors: the fluxes of resonant electrons and the anisotropy (perpendicular fluxes being larger than parallel fluxes) of electron distribution (e.g. Li et al., 2009 2010; Jiang et al., 2011). The resonant electron energy of whistlermode chorus is case-dependent but is in general on the order of $\sim 10 \mathrm{keV}$ or higher for lower-band chorus waves - the main driver of the pulsating auroras (e.g. Nishimura et al., 2010; Nishiyama et al., 2011) - in the inner magnetosphere (e.g. Ni et al., 2008). Both the chorus wave intensity and the electron anisotropy were found to peak in the inner magnetosphere $(L \leq 7)$ and tend to decrease substantially towards the outer magnetosphere (e.g. Li et al., 2009, 2010; Aryan et al., 2014). On the other hand, the energetic electron fluxes are well known to have an energy-dependent inner boundary, as known as the Alfvén layer (e.g. Jiang et al., 2011). These considerations imply that the magnetospheric origins of PPA tend to be situated around the inner boundary of a few $10 \mathrm{~s} \mathrm{keV}$ electrons in the post-midnight tail, where the interplay between the chorus waves, the flux of resonant electrons, and the electron anisotropy is more conducive to the precipitation that causes PPA.

We adopted the steady-state drift boundary model used by Jiang et al. (2011) to find the locus of the electron Alfvén layers at different electron kinetic energies and MLT sectors. For this, the Volland-Stern electric field model (Volland, 1973; Stern, 1975) is adopted,

$U(r, \phi)=-\frac{C}{r}+b r^{\gamma} \sin \phi$,

in which $\phi$ is the angle between the magnetic local time with origin at midnight, increasing counterclockwise; $C=92.4 \mathrm{kV}$ is the corotation electric potential, $\gamma$ is a shielding factor, and $b$ describes the strength of the cross-tail convection electric field, which is related to the shielding factor and the cross-polar-potential drop $(\Delta \Phi=2 b(12.5) \gamma)$. Following the findings of Jiang et al. (2011), we use a shielding factor $\gamma=3$ in this study. The cross-polar potential is parameterized by AE index according to Richmond et al. (1990).

$$
\Delta \Phi(\mathrm{kV})=22+0.119 \mathrm{AE}
$$

The variation in kinetic energy $W$ with radial distance to the Earth $L$ used in Jiang et al. (2011) is approximated by Kivelson and Southwood (1975) as follows:

$W \propto L^{-\gamma}, \gamma=2.1+0.9 \sin \alpha$,

where $\alpha$ is equatorial pitch angle of a particle. As mentioned above, the growth of the lower-band chorus is more dependent on the perpendicular flux and the particle precipitation is 
(a)

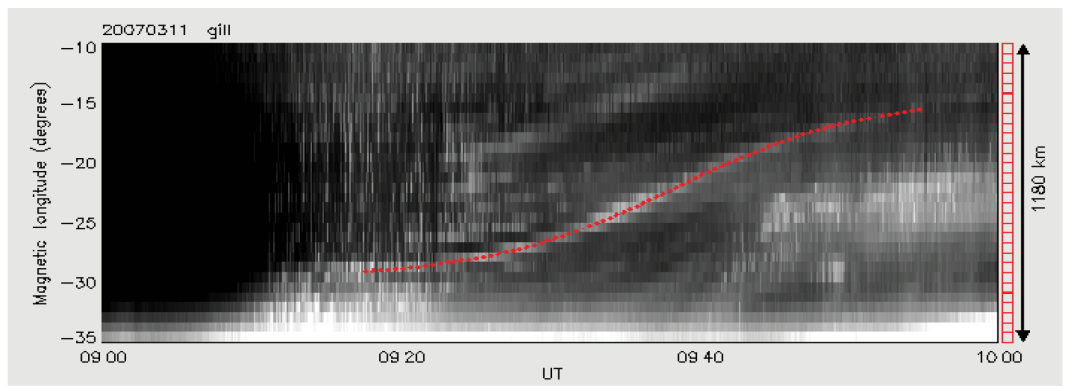

(b)

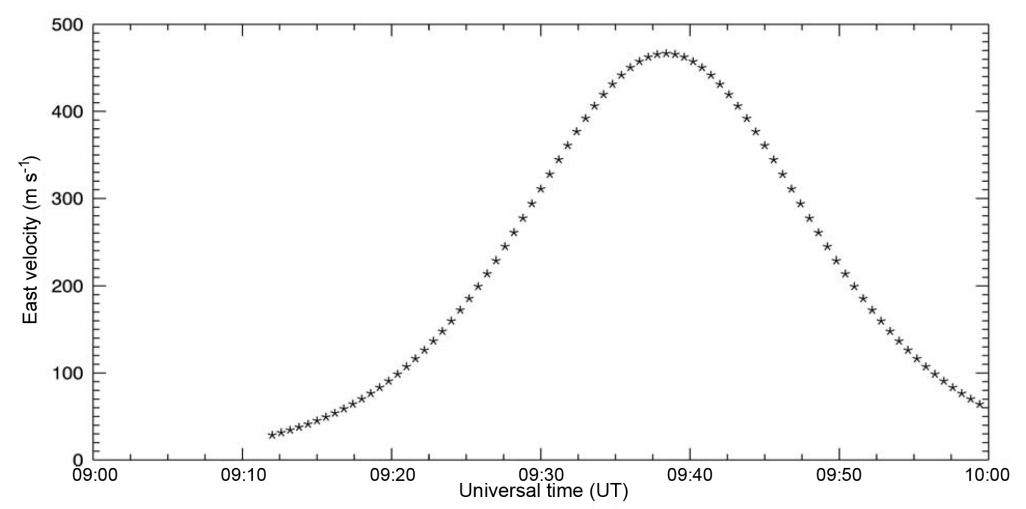

(c)

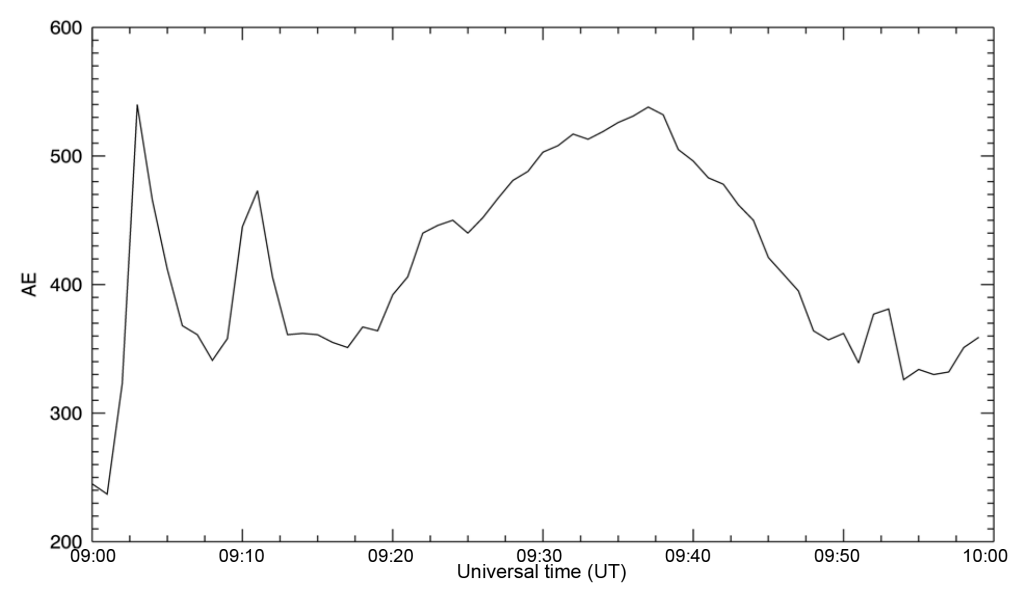

Figure 5. (a) The $1 \mathrm{~h}$ ewogram from 09:00 to 10:00 UT on 3 November 2007. The red curve is the tanh fitting result of the curve line in the ewogram. (b) Velocities derived from the fitting result in (a) with respect to universal time. (c) One-minute AE index from 09:00 to 10:00 UT.

more relevant to the parallel flux. Therefore, for demonstration purposes we choose to use a $45^{\circ}$ pitch angle in Eq. (3) in our following calculation.

Since AE value for most of the PPA events in this study is between 100 and 800, we choose to find the inner boundary of the energetic electron Alfvén layer (10 and $50 \mathrm{keV})$ on the midnight-morning sector in the equatorial plane with $\mathrm{AE}=$ 100 and $\mathrm{AE}=800$, respectively. The inner boundary of each Alfvén layer is pushed $\sim 4$ Earth radii nearer to the Earth from $\mathrm{AE}=100$ to $\mathrm{AE}=800$ (Fig. 4a).

Using the locus of the inner boundaries of 10 and $50 \mathrm{keV}$, we calculate the convection velocities along those two boundaries for $\mathrm{AE}=100$ and $\mathrm{AE}=800$ using the VollandStern electric field model. We then mapped the convection velocities into the ionosphere using the dipole magnetic field model (and the corotation velocity has been subtracted). The results are shown by the curves in Fig. 4b. As one can see, the majority of the measured velocities of PPA patches fall between the calculated convection velocities along the 10 and $50 \mathrm{keV}$ electron boundaries. This is consistent with the aforementioned notion that the magnetospheric origins of pulsating aurora, in a statistical sense, tend to be situated near the inner boundary of a few tens of keV electrons in the nightside tail.

We can see from Fig. $4 \mathrm{~b}$ that convection velocities at the inner boundary of $10 \mathrm{keV}$ plasma sheet with different $\mathrm{AE}$ are nearly the same, contrary to the findings of Weimer et al. (1990). Furthermore, the difference between convection 

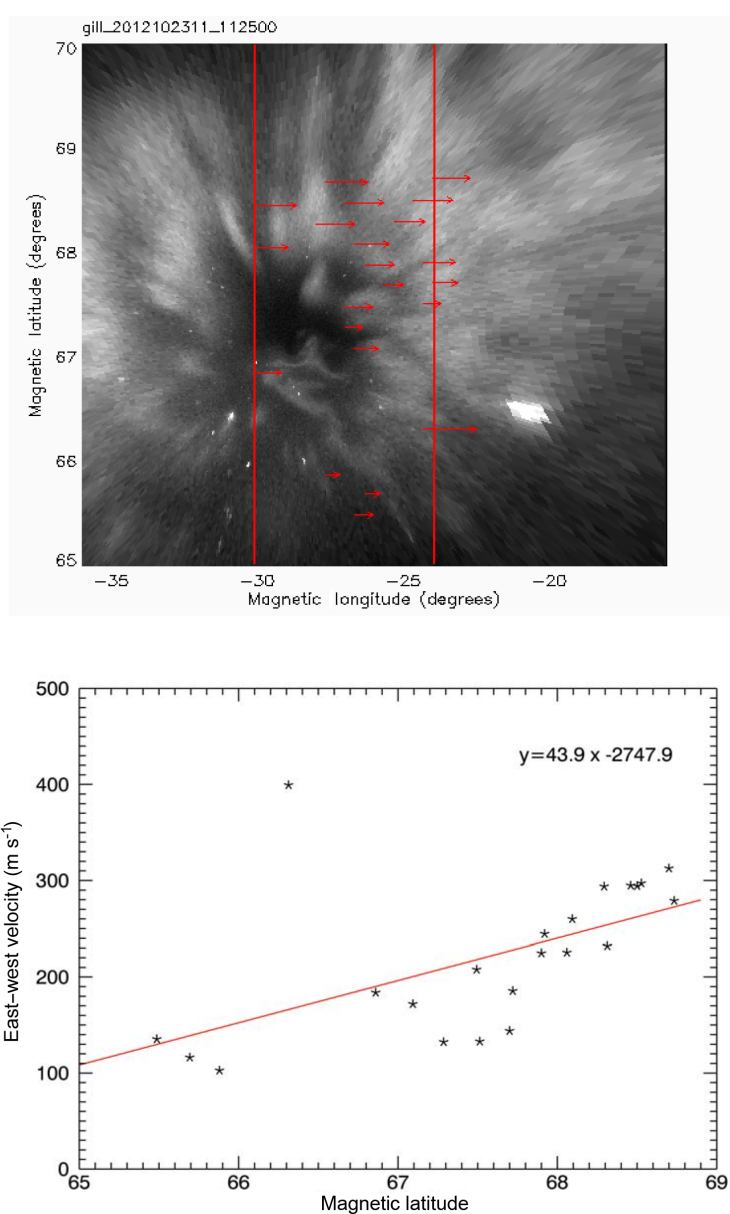

Figure 6. (a) An all-sky image at 11:25:00 UT on 23 October 2012 in geomagnetic coordinates at Gillam station. Red vectors give the velocities of the patches (longitude from $-30^{\circ}$ to $-24^{\circ}$ ) during 11:10-11:30 UT. The length of the vectors indicates the magnitude of the velocities. (b) Distribution of patch velocities with respect to magnetic latitude. The red line gives the linear fitting of all the points.

velocities at the inner boundary of $50 \mathrm{keV}$ plasma sheet with different $\mathrm{AE}$ index is small and within the uncertainty of patch velocities. As the location of electron plasma sheet shifts toward the Earth with an increasing AE index, the magnetospheric origin of PPA would also become closer to the Earth. The systematic reduction of PPA convection velocities due to the decrease in their radial distances (Fig. 4) would compete with the increase in the overall convection strength with AE index (Weimer et al., 1990). This explains why the range of PPA convection velocities, in a statistical sense, appears to be insensitive to the varying $\mathrm{AE}$ index as shown in Fig. 3c.

However, we point out that we particularly investigated $\sim 20$ events that are characterized by transient AE index variations - i.e., with timescales potentially shorter than the reconfiguration timescale of electron Alfvén layers, the con- vection velocities of all those patches are indeed observed to change with the varying AE index. Figure 5 gives an example of such a case. Figure 5a is the ewogram of a PPA patch which is observed at the Gillam station during 09:0010:00 UT on 11 March 2007. The velocity of the patch first increases and then decreases with the time. A tanh-curve model has been applied to fit the PPA trace and the fitting result is illustrated as the red dashed line in Fig. 5a. Figure $5 \mathrm{~b}$ shows the patch velocities inferred from the fitting result. Figure $5 \mathrm{c}$ gives the $1 \mathrm{~min} \mathrm{AE}$ index during the same time frame. It can be seen that, when the patch is present after 09:15 UT, the patch velocities vary in close correlation with the $\mathrm{AE}$ index in this case.

We should further mention that, in a few cases, when the AE index is quasi-stable and the PPA spans a relatively broad range of latitudes, PPA velocities are observed to decrease with the decreasing magnetic latitude. Figure 6 gives an example of such case. The event occurred during 11:1011:30 UT on 23 October 2012. We identified a total of 23 patches located within -30 to $-24^{\circ}$ magnetic longitude during this event. Figure 6a gives an image at 11:25:00 UT with east-west velocity vectors of the selected patches labeled. Note that a few patches are off and thus invisible at this epoch. As shown in Fig. 6b, patches at higher geomagnetic latitude tend to have higher eastward velocities, consistent with the general notion on the dependence of convection velocity upon latitudes in the post-midnight auroral oval.

\section{Conclusions}

Previous studies of the motion of PPA patches have led to the suggestion that their motion is governed by convection. Evidence that has been used to support this includes the fact that the size and shape of patches often persist long enough such that they cannot represent a structure in hot magnetospheric plasma, and that on a case-by-case basis the motion of individual patches is consistent with simultaneous observations of convection by ionospheric radars. However, only a few case-by-case events have been studied, and the argument based on shape has not been explored in any detail.

Here we have presented statistical results from a survey of PPA patch velocities derived from over 300 sequences of auroral images obtained by the THEMIS-ASI located at Gillam, Canada. The distribution of velocities determined in this way indicates that PPA patches mostly move westward in pre-midnight and eastward in post-midnight, respectively. We also find that, the velocities are not correlated with the value of the $\mathrm{AE}$ index in a statistical sense. The first result of direction vs. magnetic local time is consistent with the motion being convection; however, the second result is, on first consideration, at odds with that idea.

By considering the currently understood magnetospheric origin of the precipitating electrons, namely near their appropriate (based on energy) Alfvén layer, we have argued that 
the lack of dependence on AE can be reconciled with the idea of convection being the driver of patch motion. Specifically, although convection is expected to be significantly greater for higher AE than lower AE, all else being equal, it is also true that the Alfvén layer for any given (relevant) electron energy will be significantly closer to Earth for higher AE than for lower AE. Slower convection closer to Earth compensates for the larger overall convection, leading to a lack of statistical correlation between the patch motion and the AE index.

More work needs to be done on the motion of PPA patches and the evolution of their shapes. However, this study has added one piece of new evidence that supports the idea that the patch motion is convection, and another that at least does not contradict the idea. Looking forward, it is important to answer this question definitively. Its resolution, for example, will shed light on what dictates the shape of PPA patches, and will hopefully provide us a new means of remotely sensing ionospheric and hence magnetospheric convection. We suggest that while more studies of large numbers of patches will add to our confidence that the motion is indeed convection, we are in great need of a large number of simultaneous measurements of patch motion and the ionospheric convection in the same location. One possibility is to use in situ observations of the electric field from, for example, the ESA Swarm mission, but to date there have not been a significant number of Swarm flights through such structures.

\section{Data availability}

For the Patchy Pulsating Aurora (PPA) event list (.txt file), please see Supplement. All the aurora data of THEMIS ASI used in this study were downloaded from http://data.phys. ucalgary.ca/sort_by_project/THEMIS/asi/stream0/. The onehour and one-minute AE index were downloaded from the World Data Centre for Geomagnetism in Kyoto, Japan (http: //wdc.kugi.kyoto-u.ac.jp/aedir/index.html).

\section{The Supplement related to this article is available online at doi:10.5194/angeo-35-217-2017-supplement.}

Competing interests. The authors declare that they have no conflict of interest.

Acknowledgements. The THEMIS mission is supported by NASA. This study utilizes data from THEMIS-ASI sites in Canada, which are funded by the Canadian Space Agency and led by the University of Calgary.

The topical editor, E. Roussos, thanks two anonymous referees for help in evaluating this paper.

\section{References}

Angelopoulos, V.: The THEMIS mission, Space Sci. Rev., 141, doi:10.1007/s11214- 008-9336-1, 2008.

Aryan, H., Yearby, K., Balikhin, M., Agapitov, O., Krasnoselskikh, V. and Boynton, R.: Statistical study of chorus wave distributions in the inner magnetosphere using Ae and solar wind parameters, J. Geophys. Res., 119, 6131-6144, doi:10.1002/2014JA019939, 2014.

Baker, K. B. and Wing, S.: A new magnetic coordinate system for conjugate studies at high latitudes, J. Geophys. Res., 94, 91399143, doi:10.1029/JA094iA07p09139, 1989.

Bryant, D. A., Courtier, G. M., and Bennett, G.: Equatorial modulation of electrons in a pulsating aurora, Atmos. Terr. Phys., 33, 859-867, doi:10.1016/0021-9169(71)90086-9, 1971.

Davidson, G. T.: Pitch-angle diffusion and the origin of temporal and spatial structures in morningside aurorae, Space Sci. Rev, 53, 45-82, 1990.

Davis, T. N.: The morphology of the auroral displays of 1957-1958: 2. Detail analyses of Alaska data and analyses of high-latitude data, J. Geophys. Res., 67, 75-110, 1962.

Davis, T. N.: Magnetospheric convection pattern inferred from magnetic disturbance and auroral motions, J. Geophys. Res., 76, 5978-5984, 1971.

Donovan, E., Mende, S., Jackel, B., Frey, H., Syrjäsuo, M., Voronkov, I., Trondsen, T., Peticolas, L., Angelopoulos, V., Harris, S., Greffen, M., and Connors, M.: The THEMIS all-sky imaging array-system design and initial results from the prototype imager, J. Atmos. Sol.-Terr. Phys., 68, 1472-1487, 2006.

Erickson, G. M., Spiro, R. W., and Wolf, R. A.: The physics of the Harang discontinuity, J. Geophys. Res., 96, 1633-1645, doi:10.1029/90JA02344, 1991.

Greenwald, R. A., Weiss, W., Nielsen, E., and Thomson, N. R.: STARE: A new radar auroral backscatter experiment in northern Scandinavia, Radio Sci., 13, 1021-1039, doi:10.1029/RS013i006p01021, 1978.

Jiang, F., Kivelson, M. G., Walker, R. J., Khurana, K. K., Angelopoulos, V., and Hsu, T.: A statistical study of the inner edge of the electron plasma sheet and the net convection potential as a function of geomagnetic activity, J. Geophys. Res., 116, A06215, doi:10.1029/2010JA016179, 2011.

Johnstone, A. D.: Pulsating aurora, Nature, 274, 119-126, 1978.

Jones, S. L., Lessard, M. R., Rychert, K., Spanswick, E., and Donovan, E.: Large-scale aspects and temporal evolution of pulsating aurora, J. Geophys. Res., 116, A03214, doi:10.1029/2010JA015840, 2011.

Kivelson, M. G. and Southwood, D. J.: Approximations for the study of drift boundaries in the magnetosphere, J. Geophys. Res., 80, 3528-3534, doi:10.1029/JA080i025p03528, 1975.

Li, W., Thorne, R. M., Angelopoulos, V., Bortnik, J., Cully, C. M., Ni, B., LeContel, O., Roux, A., Auster, U., and Magnes, W.: Global distribution of whistler-mode chorus waves observed on the THEMIS spacecraft, Geophys. Res. Lett., 36, L09104, doi:10.1029/2009GL037595, 2009.

Li, W., Thorne, R. M., Nishimura, Y., Bortnik, J., Angelopoulos, V., McFadden, J. P., Larson, D. E., Bonnell, J. W., Le Contel, O., Roux, A., and Auster, U.: THEMIS analysis of observed equatorial electron distributions responsible for the chorus excitation, J. Geophys. Res., 115, A00F11, doi:10.1029/2009JA014845, 2010. 
Mende, S. B., Harris, S. E., Frey, H. U., Angelopoulos, V., Russell, C. T., Donovan, E., Jackel, B., Greffen, M., and Peticolas, L. M.: The THEMIS Array of Ground-based Observatories for the Study of Auroral Substorms, Space Sci. Rev., 141, 357-387, doi:10.1007/s11214-008-9380-x, 2008.

Nakamura, R. and Oguti, T.: Drifts of auroral structures and magnetospheric electric fields, J. Geophys. Res., 92, 11241-11247, doi:10.1029/JA092iA10p11241, 1987.

Ni, B., Thorne, R. M., Shprits, Y. Y., and Bortnik, J.: Resonant scattering of plasma sheet electrons by whistler-mode chorus: Contribution to diffuse auroral precipitation, Geophys. Res. Lett., 35, L11106, doi:10.1029/2008GL034032, 2008.

Nishimura, Y., Bortnik, J., Li, W., Thorne, R. M., Lyons, L. R., Angelopoulos, V., Mende, S. B., Bonnell, J. W., Le Contel, O., Cully, C., Ergun, R., and Auster, U.: Identifying the Driver of Pulsating Aurora, Science, 330, 81-84, doi:10.1126/science.1193186, 2010.

Nishiyama, T., Sakanoi, T., Miyoshi, Y., Katoh, Y., Asamura, K., Okano, S., and Hirahara, M.: The source region and its characteristic of pulsating aurora based on the Reimei observations, J. Geophys. Res., 116, A03226, doi:10.1029/2010JA015507, 2011.

Oguti, T.: Recurrent auroral patterns, J. Geophys. Res., 81, 17821786, doi:10.10291041/JA081i010p01782, 1976.

Richmond, A. D., Kamide, Y., Akasofu, S. I., Alcaydé, D., Blanc, M., de la Beaujardière, O., Evans, D. S., Foster, J. C., FriisChristensen, E., Holt, J. M., Pellinen, R. J., Senior, C., and Zaitzev, A. N.: Global measures of ionospheric electrodynamic activity inferred from combined incoherent scatter radar and ground magnetometer observations, J. Geophys. Res., 95, 10611071, doi:10.1029/JA095iA02p01061, 1990.
Royrvik, O. and Davis, T. N.: Pulsating aurora: Local and global morphology, J. Geophys. Res., 82, 4720-4740, doi:10.1029/JA082i029p04720, 1977.

Sandahl, I., Eliasson, L., and Lundin, R.: Rocket observations of precipitating electrons over a pulsating aurora, Geophys. Res. Lett., 7, 309-312, doi:10.1029/GL007i005p00309, 1980

Scourfield, M. W. J., Keys, J. G., Nielsen, E., Goertz, C. K., and Collin, H.: Evidence for the $E \times B$ drift of pulsating auroras, J. Geophys. Res., 88, 7983-7988, 1983.

Siren, J. C.: Pulsating aurora in high-latitude satillite photographs, Geophys. Res. Lett., 2, 557-560, doi:10.1029/GL002i012p00557, 1975.

Stern, D. P.: The motion of a proton in the equatorial magnetosphere, J. Geophys. Res., 80, 595-599, doi:10.1029/JA080i004p00595, 1975.

Volland, H.: A semiempirical model of large-scale magnetospheric electric fields, J. Geophys. Res., 78, 171-180, doi:10.1029/JA078i001p00171, 1973.

Weimer, D. R.: Substorm influence on the ionospheric electric potentials and currents, J. Geophys. Res., 104, 185-197, doi:10.1029/1998JA900075, 1999.

Weimer, D. R., Maynard, N. C., Burke, W. J., and Liebrecht, C.: Polar cap potentials and the auroral electrojet indices, Planet. Space Sci., 38, 1207-1222, 1990.

Yang, B., Donovan, E., Liang, J., Ruohoniemi J. M., and Spanswick, E.: Using Patchy Pulsating Aurora to Remote Sense Magnetospheric Convection, Geophys. Res. Lett., 42, 5083-5089, doi:10.1002/2015GL064700, 2015. 